\title{
ACMG Statement on Direct-to-Consumer Genetic Testing
}

\author{
American College of Medicine Genetics Board of Directors
}

Genetic tests of individuals or families for the presence of or susceptibility to disease are medical tests. At the present time, genetic testing should be provided to the public only through the services of an appropriately qualified health care professional. The health care professional should be responsible for

Approved by the Board of Directors of the American College of Medical Genetics, June 29, 2003. American College of Medicine Genetics, 9650 Rockville Pike, Bethesda, MD 20814-3998. Go to www.geneticsinmedicine.org for a printable copy of this document. DOI: 10.1097/01.GIM.0000106164.59722.CE both ordering and interpreting the genetic tests, as well as for pretest and posttest counseling of individuals and families regarding the medical significance of test results and the need, if any, for follow-up. Due to the complexities of genetic testing and counseling, the self-ordering of genetic tests by patients over the telephone or the Internet, and their use of genetic "home testing" kits, is potentially harmful. Potential harms include inappropriate test utilization, misinterpretation of test results, lack of necessary follow-up, and other adverse consequences.

Disclaimer: This guideline is designed primarily as an educational resource for medical geneticists and other health care providers to help them provide quality medical genetic services. Adherence to this guideline does not necessarily assure a successful medical outcome. This guideline should not be considered inclusive of all proper procedures and tests or exclusive of other procedures and tests that are reasonably directed to obtaining the same results. In determining the propriety of any specific procedure or test, the geneticist should apply his or her own professional judgment to the specific clinical circumstances presented by the individual patient or specimen. It may be prudent, however, to document in the patient's record the rationale for any significant deviation from this guideline. 